\title{
Assessment of levels of the tumor markers HE4 and CA1 25 considering staging, grading and histological types of endometrial cancer
}

\author{
Nabil Abdalla, Robert Piórkowski, Paweł Stanirowski, Anna Słomka, Krzysztof Cendrowski, \\ Włodzimierz Sawicki
}

Chair and Clinic of Obstetrics, Gynecology and Oncology, Second Faculty of Medicine, Medical University of Warsaw, Poland

\begin{abstract}
Aim of the study was to assess statistical differences of serum levels of HE4 and CA125 between certain endometrial cancer stages, grading and histological types.

Material and methods: A retrospective study of 52 patients admitted to our clinic for a surgical operation because of endometrial cancer was performed. HE4 and CA125 were measured for each patient. The staging was done according to FIGO. The statistical difference of serum levels of tumor markers was analyzed considering different stages, grading and histological types.

Results: Most of the patients (92.31\%) were post-menopausal. Serum levels of tumor markers were significantly higher among patients with stage IB-IIIC than stage IA, among patients with stages II-III than stage I and among patients with stage IIIC than stage IA-IIIB. Only HE4 was significantly higher among patients with stage IB than stage IA and among patients with grading $\mathrm{G} 2$ and $\mathrm{G} 3$ than those with $\mathrm{G} 1$. Only CA125 was significantly higher among patients with stage IIIA and IIIB than those with stages I and II. There was no statistically significant difference in level of either tumor marker in differentiation of endometrioid from other histological endometrial cancer.

Conclusions: Both tumor markers HE4 and CA125 can be useful additional tools for pre-surgical differentiation between different stages of endometrial cancer. HE4 can predict advanced histological grades. Neither HE4 nor CA125 can differentiate endometrioid from other histological types of endometrial cancer.
\end{abstract}

Key words: endometrial cancer, CA125, HE4, staging, grading.

\section{Introduction}

Endometrial cancer (EC) is the fourth most common cancer among women in Poland and constitutes $7 \%$ of all malignancies [1]. Endometrial cancer can be subdivided into two main types: I and II. Type I EC constitutes $80 \%$ of all cases of EC and is related to long duration of unopposed estrogen stimulation. Type II EC patients are diagnosed with high-risk histological types (serous, clear cell and high-grade endometrioid) and constitute about $20 \%$ of all patients with EC [2,3]. Most EC cases are diagnosed in earlier stages due to postmenopausal bleeding $[4,5]$. The prognosis is good since the minority of cases $(25 \%)$ present with metastases at the time of initial diagnosis [4]. The prognosis is strongly related to stage, and the survival rate is about $90 \%$ for stage I disease [6].

Clinical staging of EC was replaced by surgical in 1988, as clinical assessment of staging of EC failed in $20 \%$ of cases $[7,8]$. The staging revised by the International Federation of Gynecology and Obstetrics (FIGO) includes assessment of the pelvic and para-aortic lymph nodes. However, the need for pelvic and/or para-aortic lymphadenectomy in the management of EC remains controversial. Lymphadenectomy is associated with lower limb edema, lymph cyst formation, increased operating time and need of a specialized oncologist [7].

Due to the morbidity associated with lymphadenectomy, imaging techniques and tumor markers have been investigated to replace lymphadenectomy with non-surgical techniques that can correctly select patients for the optimum method of management [9]. In the study of Alcazar et al. the sensitivity and specificity of transvaginal/transrectal ultrasound for identifying high-risk cases according to myometrial infiltration, cervical involvement and adnexal involvement were $78.0 \%$ and $89.1 \%$ respectively [10]. The study of Kim et al. showed that the sensitivity of positron emission tomography/computed tomography (PET/CT) and magnetic resonance imaging (MRI) for detecting metastatic lymph nodes was $70 \%$ and $34 \%$ respectively, while the specificity was $95.4 \%$ and $95 \%$ respectively [11]. San- 
juan et al. revealed that the sensitivity of intraoperative frozen biopsy for histological grading and myometrial invasion was $40 \%$ and $74 \%$ respectively, while the specificity was $98 \%$ and $95 \%$ respectively [12].

CA125 was first detected by Bast et al. as epithelial ovarian cancer antigen [13]. The role of CA125 in the diagnosis, staging and prognosis of EC has been widely investigated, but it can be elevated in many gynecological and non-gynecological diseases, and as of now it is not routinely used in the management of EC $[14,15]$.

HE4 was detected for the first time in the epithelium of distal epididymis [16]. Recently, HE4 was found to be overexpressed in EC and may have some pathophysiological functions. The study of Lu et al. showed that management of EC cell lines with purified, extracellular HE4 led to a significant increase in cell viability, proliferation, and DNA synthesis. This function may contribute to EC progression and/or metastases [17]. Recent studies in the literature showed the importance of this novel marker in the diagnosis, staging and prognosis of EC. Capriglione et al. for the first time analyzed the correlation between the preoperative serum level of HE4 and EC staging and grading [18].

In our study we present our experience of assessment of both tumor markers HE4 and CA125 in the pre-surgical prediction of EC staging, grading and histological types.

The aim of the study was to assess the statistical differences of pre-surgical serum levels of tumor markers HE4 and CA125 between certain stages, histological types and classes of histological grading of EC.

\section{Material and methods}

All patients with EC managed at our clinic between October 2012 and June 2015 were reviewed and considered for study recruitment. The inclusion criteria were as follows: age above 18 years old, histological diagnosis of EC, complete staging of the patients according to FIGO and documented serum levels of HE4 and CA125 measured within one week before the operation. Patients with personal history of another malignancy and renal disease were excluded from the study. Both HE4 and Ca125 were measured at the same time with kits (Fujirebio Diagnostics, Inc.) in the same apparatus, Cobas 8000 e602, using electro-chemiluminescence immunoassay (ECLIA). The WHO histological classification of EC was used in the study. The histological types were divided into two main types: endometrioid adenocarcinoma and other non-endometrioid subtypes. As reported in the literature, endometrial histological grading whether mild G1, moderate $G 2$ or severe $G 3$ can be applied only to endometrioid EC [3]. Endometrioid EC was taken in the statistical analysis considering the histological grading. Menopause was defined as absence of menstruation for at least one year. The description analysis of distribution of patients regarding menopause, histological type, staging and histological grading was performed. The Shapiro-Wilk (S-W) test was used to evaluate the distribution of the variables of HE4 and CA125. The nonparametric Mann-Whitney $U$ test was used to determine the difference of serum concentration of tumor markers HE4 and CA125 between certain stages, grading and histological

Tab. I. Comparison of different groups regarding levels of HE4 and CA125

\begin{tabular}{|c|c|c|c|c|c|c|}
\hline \multirow{2}{*}{$\begin{array}{l}\text { Comparison } \\
\text { between groups }\end{array}$} & \multirow{2}{*}{ Groups compared } & \multirow{2}{*}{$\begin{array}{l}\text { Number of patients } \\
\text { (percent of the total) }\end{array}$} & \multicolumn{2}{|c|}{ HE4 } & \multicolumn{2}{|c|}{ CA125 } \\
\hline & & & Median & $p$ value & Median & $p$ value \\
\hline \multirow{2}{*}{ IB-IIIC vs. IA } & IA & $19(36.5)$ & 73.20 & \multirow{2}{*}{$<0.001$} & 12.82 & \multirow{2}{*}{0.001} \\
\hline & IB-IIIC & $33(63.4)$ & 129.50 & & 27.08 & \\
\hline \multirow{2}{*}{ IB vs. IA } & IA & $19(52.8)$ & 73.20 & \multirow{2}{*}{$<0.001$} & 12.82 & \multirow{2}{*}{0.076} \\
\hline & IB & $17(47.2)$ & 123.50 & & 22.30 & \\
\hline \multirow{2}{*}{ II and III vs. I } & I & $36(69.2)$ & 89.70 & \multirow{2}{*}{0.009} & 15.72 & \multirow{2}{*}{$<0.001$} \\
\hline & II and III & $16(30.7)$ & 175.15 & & 66.03 & \\
\hline \multirow{2}{*}{$\begin{array}{l}\text { IIIA and IIIB } \\
\text { vs. I and II }\end{array}$} & I and II & $40(81.6)$ & 91.70 & \multirow{2}{*}{0.086} & 15.80 & \multirow{2}{*}{0.006} \\
\hline & IIIA and IIIB & $9(18.3)$ & 173.70 & & 54.00 & \\
\hline \multirow{3}{*}{$\mathrm{G} 1, \mathrm{G} 2, \mathrm{G} 3$} & G1 & $34(73.9)$ & 91.70 & \multirow{3}{*}{0.054} & 16.11 & \multirow{3}{*}{0.293} \\
\hline & G2 & $9(19.5)$ & 120.00 & & 22.30 & \\
\hline & G3 & $3(6.5)$ & 213.00 & & 96.30 & \\
\hline \multirow{2}{*}{$\mathrm{G} 3$ and $\mathrm{G} 2$ vs. G1 } & G1 & $34(73.9)$ & 91.70 & \multirow{2}{*}{0.020} & 16.11 & \multirow{2}{*}{0.211} \\
\hline & G2 and G3 & $12(26.0)$ & 120.50 & & 25.01 & \\
\hline \multirow{2}{*}{ IIIC vs. IA-IIIB } & $|A-||| B$ & $49(94.2)$ & 96.00 & \multirow{2}{*}{0.011} & 18.09 & \multirow{2}{*}{0.014} \\
\hline & IIIC & $3(5.7)$ & 368.60 & & 193.60 & \\
\hline \multirow{2}{*}{$\begin{array}{l}\text { Endometrioid vs. other } \\
\text { histological types }\end{array}$} & Endometrioid & $46(88.4)$ & 99.35 & \multirow{2}{*}{0.318} & 18.09 & \multirow{2}{*}{0.195} \\
\hline & Other types & $6(11.5)$ & 73.85 & & 22.29 & \\
\hline
\end{tabular}


types. A $p$ value of less than 0.05 was considered as significant in the statistical analysis.

\section{Results}

The retrospective study included 52 patients with EC. The age range was 37 to 86 years, with a mean of 66.5 years (standard deviation [SD] $=10.6$ years). Menopause was noted in $48(92.31 \%)$ of patients. A total of $46(88.46 \%)$ patients were diagnosed with endometrioid EC, while 4 (7.69\%) and 2 (3.85\%) patients were diagnosed with serous and clear cell carcinoma subtypes respectively. A total of 36 (69.23\%), 4 (7.69\%) and 12 (23.07\%) patients were diagnosed at stage I, II and III respectively. Stage I cases included 19 (36.53\%) and 17 (32.69\%) patients with stage IA and IB respectively. Within stage III patients, a total of 3 (5.76\%), 6 (11.53\%) and 3 (5.76\%) had stage IIIA, IIIB and IIIC respectively. Histological grade G1, G2 and G3 of endometrioid EC was diagnosed in 34 (73.91\%), 9 (19.56\%) and 3 (6.52\%) patients respectively. The mean level of HE4 and CA125 was 162.2 (SD 227.7) $\mathrm{pmol} / \mathrm{l}$ and 72.58 (SD 178.47) $\mathrm{U} / \mathrm{ml}$ respectively. The significance level for the S-W test was $<0.001$ for both markers. Mann-Whitney $U$ and Kruskal- Wallis tests were used for determining the statistical difference of serum concentrations of tumor markers CA125 and HE4 between certain groups considering histological type, staging and histological grading. The results are shown in Table I.

Serum levels of HE4 and CA125 were significantly higher among patients with stage IB-IIIC than stage IA. Similarly, the serum levels of both markers were significantly higher among patients with stages II-III than patients with stage I. Only the serum level of HE4 was significantly higher among patients with stage IB than those with stage IA. HE4 level was also significantly higher among patients with higher grades (G2 and G3) than those with $\mathrm{G} 1$. The serum level of HE4 and CA125 was significantly higher among patients with stage IIIC than those with lower stages.

The results of sensitivity and specificity for HE4 at a cut-off level of $70 \mathrm{pmol} / \mathrm{l}$ and for CA125 at a cut-off level of $35 \mathrm{U} / \mathrm{ml}$ and $20 \mathrm{U} / \mathrm{ml}$ regarding the differentiation between different groups of patients are shown in Table II.

Tumor marker Ca125 at a cut-off level of $35 \mathrm{U} / \mathrm{ml}$ has the best sensitivity and specificity for differentiation of IA-IIIB stages from IIIC stage of EC. The marker CA125 at cut-off levels of $35 \mathrm{U} / \mathrm{ml}$ and $20 \mathrm{U} / \mathrm{ml}$ has higher specific- ity than HE4 at a cut-off level of $70 \mathrm{pmol} / \mathrm{l}$ in differentiation of stages IA from IB-IIIC, in differentiation of stage I from stages ||-III and in differentiation of stages IA-IIIB from IIIC.

\section{Discussion}

The serum levels of HE4 and CA125 were not significantly higher among patients with endometrioid than among other histological subtypes of EC. These findings are supported by the previous findings in the literature [19-21]. The serum levels of tumor markers may not predict the histological subtypes of EC. However, the results of Angioli et al. showed a statistically significant different level of HE4 between endometrioid and non-endometrioid EC [22]. Further prospective studies are necessary to support our findings.

Neither HE4 nor CA125 correlated significantly with the histological grading of EC in the whole group of studied patients. However, HE4 is significantly higher among patients with advanced histological grading (G2 and G3) than those with $\mathrm{G} 1(p=0.02)$. Similar results were obtained in the study of Dobrzycka et al. [23].

In our study both tumor markers are significantly higher among patients with advanced stages (IB-III) than those with the earliest stage IA. These results are similar to those of the study of Minar et al. [24]. The study of Bignotti et al. revealed a significantly higher level of HE4 among patients with stages III-IV than those with stages I-II. In this study significantly higher HE4 levels were noted among patients with cervical involvement than those without, and among patients with adnexal involvement than those without [20]. Advanced age is a risk factor for recurrence of EC. Angioli et al. observed a significant difference between HE4 values at the primary diagnosis, comparing recurrent and non-recurrent patients, while the difference of CA125 level between these groups was not statistically significant [2].

In our study the patients with positive lymph node metastases had a 10.7 times higher median level of CA125 than those without metastases, while HE4 was only 3.8 times higher. The serum concentrations of both tumor markers were significantly higher among patients with lymph node metastases than those without. One explanation why tumor markers are significantly higher when there are lymph node metastases is that when the tumor is localized to the uterus there is less access to the blood

Tab. II. Results of sensitivity and specificity of HE4 and CA125 regarding differentiation between certain groups of patients

\begin{tabular}{|c|c|c|c|c|c|c|}
\hline $\begin{array}{l}\text { Group } \\
\text { comparison }\end{array}$ & $\begin{array}{l}\text { Sensitivity of HE4 } \\
\text { at cut-off level } \\
\text { of } 70 \mathrm{pmol} / \mathrm{l}\end{array}$ & $\begin{array}{l}\text { Specificity of HE4 } \\
\text { at cut-off level } \\
\text { of } 70 \mathrm{pmol} / \mathrm{l}\end{array}$ & $\begin{array}{l}\text { Sensitivity of } \\
\text { CA125 at cut-off } \\
\text { level of } 35 \mathrm{U} / \mathrm{ml}\end{array}$ & $\begin{array}{c}\text { Specificity of } \\
\text { CA125 at cut-off } \\
\text { level of } 35 \mathrm{U} / \mathrm{ml}\end{array}$ & $\begin{array}{l}\text { Sensitivity of } \\
\text { CA125 at cut-off } \\
\text { level of } 20 \mathrm{U} / \mathrm{ml}\end{array}$ & $\begin{array}{c}\text { Specificity of } \\
\text { CA125 at cut-off } \\
\text { level of } 20 \mathrm{U} / \mathrm{ml}\end{array}$ \\
\hline Ia vs. IB-IIIC & 84.8 & 47.4 & 43.8 & 94.7 & 71.9 & 89.5 \\
\hline | vs. II-III & 81.3 & 30.6 & 60.0 & 83.3 & 86.7 & 66.7 \\
\hline$|A-I| \mid B$ vs. IIIC & 100.0 & 28.6 & 100.0 & 75.0 & 100.0 & 54.2 \\
\hline
\end{tabular}


circulation or lymphatics [21]. Our findings are supported by the previous studies $[20,25,26]$. In the study of Koper et al., the sensitivity to identify patients who would have required a lymphadenectomy or sampling on the basis of pre-curettage serum CA125 measurements ranged between $17 \%$ with a cut-off of $35 \mathrm{U} / \mathrm{ml}$, and $53 \%$ with a cut-off of $15 \mathrm{U} / \mathrm{ml}$. Corresponding specificity to exclude patients for lymphadenectomy or sampling ranged from $95 \%$ to $76 \%$ respectively [27]. In the study of Antonsen et al., the sensitivity and specificity of HE4 at a cut-off of $70 \mathrm{pmol} / \mathrm{l}$ to detect lymph node metastases was $75.9 \%$ and $48.8 \%$ respectively, while for CA125 at a cut-off level of $35 \mathrm{U} / \mathrm{ml}$ the respective values were $62.1 \%$ and $78.9 \%$ [26].

The serum level of HE4 is not only elevated in cases of distant metastases. It was significantly higher among patients with stage IB than those with stage IA $(p \leq 0.001)$. In this situation the higher levels of HE4 are related to greater depth of invasion in the myometrium, which can be related to more cancerous cell involvement in the secretion of HE4 [5]. At the same time we did not find this significant difference considering CA125 ( $p=0.076)$. Our results are supported by the results of Minar et al. [24]. Kalogera et al. observed a significantly higher level of HE4 among patients with type I EC with more than $50 \%$ myometrial invasion than those with $50 \%$ or less invasion of the myometrium. Here the serum level of HE4 was also significantly correlated with tumor size, and it was significantly higher among patients with the primary tumor diameter greater than $2 \mathrm{~cm}$ than those with the diameter equal to or less than $2 \mathrm{~cm}$ [28].

In the study of Li et al., the immunohistochemistry method was used to determine expression of HE4 in 102 cases of EC tissues. The results showed increasing expression of HE4 with increasing clinical stage. There was significantly higher expression of HE4 among patients with stage III-IV than those with stage I-II [5]. The serum level of HE4 in our study was not significantly different between the group of patients with stage I-II and the group of stage IIIA-IIIB. This might be related to the lower number of patients in our study or to a possible weak association between HE4 tissue expression and serum HE4 level at an advanced stage. Li et al. also reported that with a decrease in the degree of differentiation of EC, the positive expression rate of HE4 was increased significantly. The endometrial tissue expression of HE4 was not significantly higher among patients with lymph node metastases than those without metastases. However, a significant difference was found between these groups of patients considering the strong positive expression rate of HE4 [5].

A significantly higher level of CA125 was observed in the study of Yildiz et al. among patients with advanced stages of EC than in stage I. A significantly higher level of CA125 was observed among patients with lymph node metastases than among those without [25]. These results are compatible with our results.
Nicklin et al. observed a significantly higher level of CA125 among patients with an advanced stage in comparison to stage I EC patients. In this study, at a cut-off level of $30 \mathrm{U} / \mathrm{ml}$, the sensitivity, specificity, and positive and negative predictive values for detecting extrauterine disease were $34.2 \%, 87 \%, 43.1 \%$ and $82.1 \%$ respectively, while the sensitivity, specificity, and positive and negative predictive values for detecting nodal involvement were $54.8 \%, 87.2 \%, 17.35 \%$ and $97.5 \%$ respectively [29].

Chung et al. reported that a higher CA125 level was significantly correlated with advanced stage, myometrial invasion and lymph node metastases [30]. Sood et al. found that a higher level of CA125 (> $35 \mathrm{U} / \mathrm{ml})$ was associated with higher stages, advanced grading, myometrial invasion and lymph node involvement. Preoperative CA125 level greater than $65 \mathrm{U} / \mathrm{ml}$ was the most significant predictor of extra-uterine disease and carried a 6.5 -fold higher risk. Patients with a preoperative CA125 level less than or equal to $20 \mathrm{U} / \mathrm{ml}$ might be considered as candidates for vaginal hysterectomy unless unfavorable histology or a high-grade (grade II or III) tumor is present. This approach eliminated $24 \%$ of the abdominal staging procedures, with a risk of less than 3\% for extra-uterine disease [31].

In the study of Hsiech et al. a significantly higher level of CA125 was significantly correlated with surgical stage of EC, myometrial invasion, cervical invasion, adnexal involvement, positive washing cytology, pelvic and/or para-aortic lymph node metastases and distant metastases. Data of this study provided evidence that a preoperative level of CA125 more than $40 \mathrm{U} / \mathrm{ml}$ can be a criterion in selecting patients for full pelvic lymphadenectomy [21].

Our study has some limitations. A total of 52 patients were enrolled in the single-center retrospective study with only 6 cases of clear cell and serous subtypes of EC. The different number of patients in different stages of endometrial cancer together with low specificity of HE4 may affect our preliminary results. The strengths of our study include the fact that serum levels of HE4 and CA125 were measured within one week before surgical staging using the same protocol. Patients with renal diseases were excluded from the study as previous studies showed that the HE4 level is increased in renal diseases. In the study of Bolstad et al., higher HE4 levels were associated with increased creatinine levels. If a creatinine level of $50 \mathrm{nmol} / \mathrm{l}$ is considered baseline, $70 \mathrm{nmol} / \mathrm{l}$ is associated with $12 \%$ higher levels and $90 \mathrm{nmol} / \mathrm{l}$ with $22 \%$ higher levels [32].

In this study we adopted the staging protocol of FIGO 2009. Stage IA according to this protocol includes patients with EC involving the endometrium with/without infiltration of the myometrium, with maximum infiltration of less than $50 \%$ of the uterine wall. Further prospective studies are needed with consideration of 
level of both tumor markers in patients with localized EC with/without infiltration of the myometrium. This might help to identify those patients with EC confirmed only for an endometrial polyp or the endometrium, where curettage or therapeutic hysteroscopic removal of the pathological changes may be adequate. Identification of localized EC is very important for younger patients, where fertility-preserving treatment might be a choice for them [28].

\section{Conclusions}

The following conclusions are based on our preliminary results:

1. The serum levels of HE4 and CA125 cannot predict the histological differentiation of endometrioid from other histological subtypes of endometrial cancer.

2. Both tumor markers can be used for differentiation of advanced stages of endometrial cancer from the earliest stage IA.

3. HE4, in contrast to CA125, can be used to differentiate stage IB from stage IA of endometrial cancer and in differentiation of aggressive histological grading classes (G2 and G3) from the mild form G1.

\section{Acknowledgements}

This study was supported by grant project of Medical University of Warsaw for research and scientific work aimed at scientific development of young doctors and PhD students. (Nr 2WA/PM22D/15).

\section{Disclosure}

\section{Authors report no conflict of interest.}

\section{References}

1. Didkowska J, Wojciechowska U. Zachorowania i zgony na nowotwory złośliwe w Polsce. Krajowy Rejestr Nowotworów, Centrum Onkologii Instytut im. Marii Skłodowskiej-Curie. http://onkologia.org.pl/k/epidemiologia/ dostęp z dnia 18/04/2016.

2. Angioli R, Capriglione S, Scaletta G, et al. The role of HE4 in endometrial cancer recurrence: how to choose the optimal follow-up program. Tumour Biol 2016; 37: 4973-4978.

3. Amant F, Moerman P, Neven P, et al. Endometrial cancer. Lancet 2005; 366: 491-505.

4. Saarelainen SK, Peltonen N, Lehtimäki T, et al. Predictive value of serum human epididymis protein 4 and cancer antigen 125 concentrations in endometrial carcinoma. Am J Obstet Gynecol 2013; 209: 142.e1-6.

5. Li X, Gao Y, Tan M, et al. Expression of HE4 in endometrial cancer and its clinical significance. Biomed Res Int 2015; 2015: 437468.

6. Bie Y, Zhang Z. Diagnostic value of serum HE4 in endometrial cancer: a meta-analysis. World I Surg Oncol 2014; 12: 169

7. Jiang T, Huang L, Zhang S. Preoperative serum CA125: a useful marker for surgical management of endometrial cancer. BMC Cancer 2015; 15: 396

8. Capriglione S, Plotti F, Miranda A, et al. Utility of tumor marker HE4 as prognostic factor in endometrial cancer: a single-center controlled study. Tumour Biol 2015; 36: 4151-4156.

9. Meissnitzer M, Meissnitzer T, Forstner R. Gynecological tumors of the true pelvis: Radiological diagnosis for pelvic tumors made simple. Radiologe 2015; 55: 1117-1132.
10. Alcázar JL, Pineda L, Caparrós M, et al. Transvaginal/transrectal ultrasound for preoperative identification of high-risk cases in well or moderately differentiated endometrioid carcinoma of the endometrium. Ultrasound Obstet Gynecol 2016; 47: 374-379.

11. Kim HJ, Cho A, Yun M, et al. Comparison of FDG PET/CT and MRI in lymph node staging of endometrial cancer. Ann Nucl Med 2016; 30: 104-113.

12. Sanjuán A, Cobo T, Pahisa J, et al. Preoperative and intraoperative assessment of myometrial invasion and histologic grade in endometrial cancer: role of magnetic resonance imaging and frozen section. Int J Gynecol Cancer 2006; 16: 385-390.

13. Bast RC Jr, Klug TL, St John E, et al. A radioimmunoassay using a monoclonal antibody to monitor the course of epithelial ovarian cancer. N Engl J Med 1983; 309: 883-887.

14. Kim HS, Park CY, Lee JM, et al. Evaluation of serum CA-125 levels for preoperative counseling in endometrioid endometrial cancer: a multicenter study. Gynecol Oncol 2010; 118: 283-288.

15. Escudero JM, Auge JM, Filella X, et al. Comparison of serum human epididymis protein 4 with cancer antigen 125 as a tumor marker in patients with malignant and nonmalignant diseases. Clin Chem 2011; 57 : 1534-1544.

16. Kirchhoff C, Habben I, Ivell R, Krull N. A major human epididymis-specific CDNA encodes a proteinwith sequence homology to extracellular proteinase inhibitors. Biol Reprod 1991; 45: 350-357.

17. Lu Q, Chen H, Senkowski C, et al. Recombinant HE4 protein promotes proliferation of pancreatic and endometrial cancer cell lines. Oncol Rep 2016; 35: 163-170.

18. Capriglione S, Plotti F, Miranda A, et al. Utility of tumor marker HE4 as prognostic factor in endometrial cancer: a single-center controlled study. Tumour Biol 2015; 36: 4151-4156.

19. Brennan DJ, Hackethal A, Metcalf AM, et al. Serum HE4 as a prognostic marker in endometrial cancer - a population based study. Gynecol Oncol 2014; 132: 159-165.

20. Bignotti E, Ragnoli M, Zanotti L, et al. Diagnostic and prognostic impact of serum HE4 detection in endometrial carcinoma patients. Br J Cancer 2011; 104: 1418-1425.

21. Hsieh CH, ChangChien CC, Lin H, et al. Can a preoperative CA 125 level be a criterion for full pelvic lymphadenectomy in surgical staging of endometrial cancer? Gynecol Oncol 2002; 86: 28-33.

22. Angioli R, Plotti F, Capriglione S, et al. The role of novel biomarker HE4 in endometrial cancer: a case control prospective study. Tumour Biol 2013; 34: 571-576.

23. Dobrzycka B, Mackowiak-Matejczyk B, Terlikowska KM, et al. Utility of HE4 to identify patients with endometrioid endometrial cancer who may require lymphadenectomy. Adv Med Sci 2015; 61: 23-27.

24. Minar L, Klabenesova I, Jandakova E, et al. Prognostic value of human epididymis protein 4 in endometrial cancer and its utility for surgical staging. J Obstet Gynaecol Res 2015; 41: 1644-1652.

25. Yildiz A, Yetimalar H, Kasap B, et al. Preoperative serum CA 125 level in the prediction of the stage of disease in endometrial carcinoma. Eur J Obstet Gynecol Reprod Biol 2012; 164: 191-195.

26. Antonsen SL, Høgdall E, Christensen IJ, et al. HE4 and CA125 levels in the preoperative assessment of endometrial cancer patients: a prospective multicenter study (ENDOMET). Acta Obstet Gynecol Scand 2013; 92: 1313-1322.

27. Koper NP, Massuger LF, Thomas CM, et al. Serum CA 125 measurements to identify patients with endometrial cancer who require lymphadenectomy. Anticancer Res 1998; 18: 1897-1902.

28. Kalogera E, Scholler N, Powless C, et al. Correlation of serum HE4 with tumor size and myometrial invasion in endometrial cancer. Gynecol Oncol 2012; 124: 270-275.

29. Nicklin J, Janda M, Gebski V, et al. The utility of serum CA-125 in predicting extra-uterine disease in apparent early-stage endometrial cancer. Int J Cancer 2012; 131: 885-890.

30. Chung HH, Kim JW, Park NH, et al. Use of preoperative serum CA-125 levels for prediction of lymph node metastasis and prognosis in endometrial cancer. Acta Obstet Gynecol Scand 2006; 85: 1501-1505.

31. Sood AK, Buller RE, Burger RA, et al. Value of preoperative CA 125 level in the management of uterine cancer and prediction of clinical outcome. Obstet Gynecol 1997; 90: 441-447.

32. Bolstad N, Øijordsbakken M, Nustad K, Bjerner J. Human epididymis protein 4 reference limits and natural variation in a Nordic reference population. Tumour Biol 2012; 33: 141-148. 